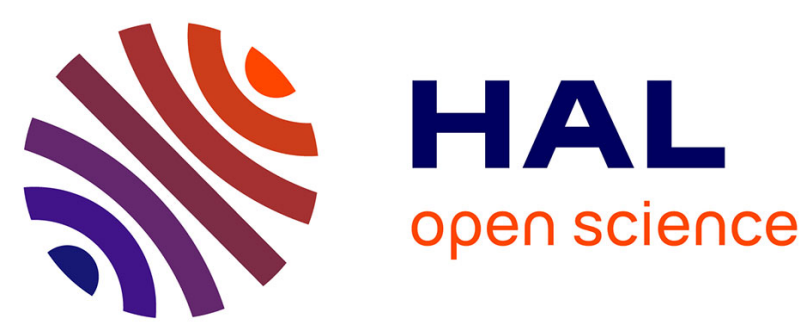

\title{
CARACTÉRISATION DE MILIEUX PLANS STRATIFIÉS À PROFIL LINÉAIRE PAR MESURE DE LA CÉLÉRITÉ D'ONDES DE STONELEY-SCHOLTE. ÉTUDE D'UNE SIMULATION
}

\author{
J. Pouliquen, A. Defebvre, L. Moukala
}

\section{To cite this version:}

J. Pouliquen, A. Defebvre, L. Moukala. CARACTÉRISATION DE MILIEUX PLANS STRATIFIÉS À PROFIL LINÉAIRE PAR MESURE DE LA CÉLÉRITÉ D'ONDES DE STONELEY-SCHOLTE. ÉTUDE D'UNE SIMULATION. Journal de Physique IV Proceedings, 1992, 02 (C1), pp.C1-861-C1864. 10.1051/jp4:19921188 . jpa-00251150

\author{
HAL Id: jpa-00251150 \\ https://hal.science/jpa-00251150
}

Submitted on 1 Jan 1992

HAL is a multi-disciplinary open access archive for the deposit and dissemination of scientific research documents, whether they are published or not. The documents may come from teaching and research institutions in France or abroad, or from public or private research centers.
L'archive ouverte pluridisciplinaire HAL, est destinée au dépôt et à la diffusion de documents scientifiques de niveau recherche, publiés ou non, émanant des établissements d'enseignement et de recherche français ou étrangers, des laboratoires publics ou privés. 


\title{
CARACTÉRISATION DE MILIEUX PLANS STRATIFItes À PROFIL LINEAIRE PAR MESURE de LA CÉLÉRITÉ D’ONDES DE STONELEY-SCHOLTE. ÉTUDE D'UNE SIMULATION
}

\author{
J. POULIQUEN, A. DEFEBVRE et L.M. MOUKALA \\ Faculté Libre des Sciences, Laboratoire d'Acoustique-Ultrasons, UA 253 CNRS, 13 rue de Toul, \\ F-59046 Lille cedex, France
}

\begin{abstract}
The conjugate-gradient numerical method is used to derive longitudinal and transverse bulk waves celerities distributions according to the depth in a PVC, from discrete measurements of Stoneley-Sholte wave celerity performed on a $\mathrm{OH}_{2} / \mathrm{PVC} / \mathrm{Al}$ system. As those distributions are linear with depth some particular procedures are allowed and we compare their differing results.
\end{abstract}

\section{INTRODUCTION}

Nous avons antéricurement mené des travaux d'évaluation non destructive de milieux stratifiés solides à couche métallique discrète (acier chromé [1]) ou à gradient de propriétés (verres trempés [2]) à partir de mesures de dispersion de célérité d'ondes de Rayleigh (OR).

Nous visons ici le même objectif pour un milieu - PVC à degré de réticulation variable - présentant un profil de célérités d'ondes de volumes variant linéairement avec la profondeur, baignant dans l'eau et reposant sur un socle d'Aluminium, en partant de la dispersion de célérité d'ondes de STONELEYSCHOLTE (O.S.S.).

L'ensemble des mesures relatives à cette maquette n'étant pas établi nous procédons ici à une simulation permettant de juger la possibilité de résoudre le problème et la valeur de l'algorithme utilisé.

\section{Système étudié - Problème direct}

\section{Caractéristiques du système.}

Construit et étudié par le laboratoire de l'ICPI (Lyon), il est constitué d'une lame de PVC à réticulation variable (densité $\rho=1377 \mathrm{~kg} / \mathrm{m}^{3}$, épaisseur $E=10 \mathrm{~mm}$ ) intercalée entre un socle d'Aluminium et une couche d'eau supposés semi-infinis. Les mesures [3] établissent qu'on peut adopter pour le PVC un profil linéaire de répartition des célérités d'ondes de volume longitudinales $\mathrm{Cl}$ et transversales $C_{t}$ avec la profondeur, avec la condition $\mathrm{Cl}=2 \mathrm{C}_{t}$.

Le problème est discrétisé en modélisant la lame de $\mathrm{PVC}$ en sous-couches de nombre $\mathrm{N}$ variant de 3 à 10. S'il peut paraître logique d'attribuer aux ondes les caractéristiques mécaniques de leurs plans médians, il est préférable, en fait, d'adopter des valeurs correspondant à des points "glissants" dans les épaisseurs des couches successives, suivant la loi :

$$
\mathrm{C}_{\mathrm{t}_{\mathrm{i}}}=\mathrm{C}_{\mathrm{i}} / 2=\mathrm{A}+\mathrm{BE}(\mathrm{i}-1) /(\mathrm{N}-1) \quad \mathrm{i} \in[1, \mathrm{~N}]
$$


où i désigne le rang de la couche à compter de l'eau, avec $\mathrm{A}=884 \mathrm{~ms}^{-1} ; \mathrm{B}=35,5 \mathrm{~ms}^{-1} \mathrm{~mm}^{-1}$ constants dans la gamme des fréquences étudiées $(50 \mathrm{kHz}<\mathrm{f}<1 \mathrm{MHz})$

Les couches extrêmes ont ainsi les caractéristiques de leurs plans supérieur ou inférieur, ce qui fournit une courbe de dispersion dont les valeurs en BF et en HF sont insensibles au choix de $\mathrm{N}$.

On adopte par ailleurs : $\mathrm{ClOH}_{2}=1496,65 \mathrm{~ms}^{-1} ; \mathrm{Cl} \mathrm{Al}=6296 \mathrm{~ms}^{-1}, \mathrm{C}_{\mathrm{t}} \mathrm{Al}=3097,1 \mathrm{~ms}^{-1}$; $\rho \mathrm{Al}=2700 \mathrm{~kg} \mathrm{~m}^{-3}$.

\section{Résolution du problème direct $[4,5]$}

Suivant des méthodes antérieurement exposées $[4,5]$ nous calculons la courbe de dispersion de célérité des OSS dont la forme (fig1) dépend du choix de $\mathbf{N}$ mais évolue entre des limites fixées par la seule première couche : célérité des ondes transversales $C_{t 1}$ de la face supérieure en BF et célérité asymptotique égale à celle des ondes de Stoneley-Scholte $C_{\$ 1}$ eau - couche supérieure en HF.

De cette courbe nous ne retiendrons qu'un certain nombre $\mathrm{Ne}_{e}\left(\mathrm{~N}_{\mathrm{e}}=8\right.$ pour un modèle à $\mathrm{N}=$ 3 couches ; $\mathrm{Ne}_{\mathrm{e}}=4$ pour $\mathrm{N}=10$ ) de valeurs régulièrement espacées jouant pour la suite le rôle de points "pseudo-expérimentaux" d'identification du matériau.

On voit (fig.1) un tel choix pour $\mathrm{Ne}_{\mathrm{e}}=8$.

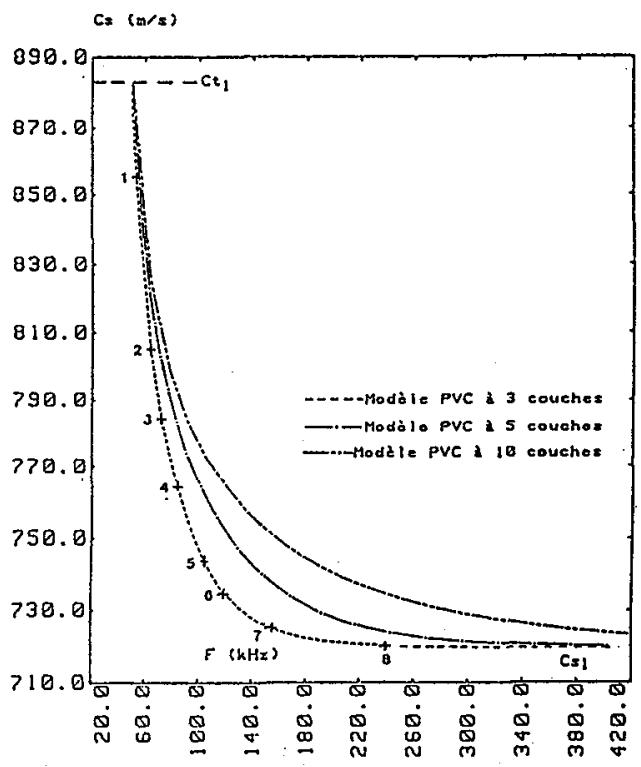

Fig.1 : Dispersion de vitesse en fonction de la fréquence

\section{Evaluation non destructive du PVC : problème inverse.}

\section{Conditions de calcul.}

La courbe de dispersion n'étant connue que par les valeurs discrètes ci-dessus définies, on cherche à retrouver les caractéristiques - célérités longitudinales $\mathrm{Cli}$ et transversales $\mathrm{C}_{\mathrm{ti}}$ - des $\mathrm{N}$ couches du modèle adopté, les épaisseurs étant fixées et la densité uniforme connue. Il y a donc au plus $2 \mathrm{~N}$ inconnues.

La recherche est menée à l'aide de l'algorithme des gradients conjugués en fixant par le choix des coefficients A et B (1) des valeurs arbitraires "de départ" aux inconnues à identifier.

Le critère d'ajustement :

$$
\mathrm{D}=\sum_{\mathrm{i}=1}^{N_{e}}\left(\mathrm{~V}_{\mathrm{ei}}-\mathrm{V}_{\mathrm{ci}}\right)^{2} / \mathrm{N}_{\mathrm{e}}
$$


écart quadratique moyen entre les vitesses "expérimentales" $V_{\text {ei }}$ et calculées $V_{c i}$ des $\mathrm{Ne}_{e}$ points d'identification, permet de juger la qualité de l'évaluation.

Les propriétés particulières du matériau nous ont incités à introduire éventuellement dans l'algorithme la contrainte (c) $\mathrm{Cl}=2 \mathrm{C}_{t}$ réduisant le nombre d'inconnues de moitié ou à imposer, de plus, en cours de calcul le respect d'un profil linéaire (cp) de vitesse avec la profondeur.

\section{Résultats obtenus.}

\subsection{Stabilité de l'algorithme.}

Adoptant au départ les valeurs vraies, celles-ci sont retrouvées à mieux que $0,5 \%$ (et souvent à quelques $10^{-4}$ près) avec des écarts $\mathrm{D}<0,2(\mathrm{~m} / \mathrm{s})^{2}$, les résultats étant meilleurs pour $\mathrm{N}=10$ couches, même si le nombre $\mathrm{Ne}_{\mathrm{e}}$ de points d'identification est réduit à 3 ou 4.

\subsection{Détermination de $A$.}

Les limites de dispersion ne dépendant que de la première couche (Cf. I 2), on identifie $A=C_{t_{1}}$ (1) en adoptant un système PVC simplifié monocouche d'épaisseur totale E. Partant de valeurs de $\mathrm{A}$ à $\pm 10 \%$ de la valeur vraie, on retrouve celle-ci à $0,01 \%$ près dans les procédures $(\mathrm{c})$ et $(\mathrm{cp})$ sous réserve de n'adopter qu'un seul point d'identification en HF proche de l'asymptote horizontale.

\subsection{Evaluation de $B$.}

Trois procédures ont été successivement utilisées en admettant $\mathrm{A}$ connu.

\subsection{Sans contrainte et sans profil.}

Une fois les valeurs initiales imposées dans (1) $\left(A=884 \mathrm{~ms}^{-1}, B=20\right.$ ou $\left.611^{3} \mathrm{~s}^{-1}\right)$ on laisse le programme évoluer dans un modèle à 3 couches $\left(2 \mathrm{~N}-1=5\right.$ variables à identifier) avec $\mathrm{N}_{\mathrm{e}}=5$ ou 8 . Les précisions sur $\mathrm{c}_{t}$ se dégradent avec la profondeur de $0,25 \%$ à $4 \% ; \mathrm{Cl}$ est médiocrement identifié et le profil n'est guère restitué.

\subsection{Avec contrainte et sans profil (c) .}

On procède en deux temps en imposant la condition $\mathrm{Cl}=2 \mathrm{Ct}$.

Estimation de $\mathrm{B}:$ Avec $\mathrm{N}=\mathrm{Ne}_{\mathrm{e}}=3, \mathrm{Bin}=2010^{3} \mathrm{~s}^{-1}$ on obtient une valeur approximative de $\mathrm{B}$, $\mathrm{B}=29,6.10^{3} \mathrm{~s}^{-1}$ à $17 \%$ près, qui servira de point de départ pour les calculs suivants.

Identification finale : Avec $\mathrm{N}=10$ ( $\mathrm{N}-1=9$ variables à identifier), on montre qu'il est avantageux de réduire $\mathrm{Ne}$ à 2 car le temps de calcul est divisé par 5,5 sans préjudice sur la précision de détermination de $\mathrm{C}_{\mathrm{t}}$ comprise entre 0,8 et $4,5 \%$ quand i croit. La restitution du profil est de qualité moyenne : l'erreur sur B étant de $6 \%\left(\mathrm{~N}_{\mathrm{e}}=4\right)$ à $9 \%\left(\mathrm{~N}_{\mathrm{e}}=2\right)$.

\subsection{Avec contrainte et avec profil (cp).}

On impose à chaque itération l'équation (1) définissant le profil pour suivre l'évolution de l'unique inconnue B par :

$$
\mathrm{B}=9\left(\mathrm{C}_{\mathrm{t} 2}-\mathrm{C}_{\mathrm{t} 1}\right) / \mathrm{E}
$$

d'où résulte par (1) la détermination temporaire des $\mathrm{Cli}_{\mathrm{i}}$ et $\mathrm{C}_{\mathrm{ti}}$.

Cette procédure autorise d'adopter des valeurs initiales de $B$ très éloignées de la réalité (de 10 à 60 ), en détermine la valeur finale de 0,15 à $0,2 \%$ près en réduisant jusqu'à $\mathrm{Ne}_{\mathrm{e}}=1$ le nombre de points d'identification avec une diminution supplémentaire d'un facteur 5 du temps de calcul et l'obtention d'écarts D négligeables. 


\section{CONCLUSION}

L'évaluation non destructive du matériau PVC de la maquette ICPI est donc possible à partir de la dispersion de célérité des OSS. Les travaux actuels s'intéressent au cas de profils linéaires distincts pour $\mathrm{Cl}_{\mathrm{l}}$ et $\mathrm{C}_{\mathrm{t}}$ avec la profondeur.

L'objectif ultime de ces travaux est de tester l'applicabilité de la méthode à l'évaluation de fonds marins [6] constitués de strates de divers sédiments ou de roches présentant des profils de célérité comparables.

\section{Références :}

[1] J.Pouliquen, A. Defebvre and B. Chenni, 12 th ICA, Proceedings vol II, 1986, G 5-2.

B. Chenni, J. Pouliquen and A. Defebvre, 13 th ICA, Proceedings vol 4, 1989, p 373-376.

[2] G.Haw, J. Pouliquen, A. Defebvre,

11 th ICA, Contributed papers, 1983, vol 2 p 223-226.

[3] Communication privée du Laboratoire de Traitement du signal ICPI-Lyon (mars 1990).

[4] A. Defebvre, J. Pouliquen, L.M. Moukala,

13 th ICA, Proceedings vol 4, 1989, p 319-322.

[5] A. Defebvre, J. Pouliquen, L.M. Moukala, Ultrasonics International 91 - à paraître.

[6] W.A. Kuperman and F.B. Fensen, Bottom Interacting Ocean Acoustics Nato conférence series - serie IV Marine Sciences. 